\title{
Effective Simulations to Support Academics in Inclusive Online Learning Design
}

\author{
George Papadopoulos \\ Accessibility Research Centre \\ University of Teesside \\ United Kingdom \\ +44 (0) 1642384648 \\ G.Papadopoulos@tees.ac.uk
}

\author{
Dr Elaine Pearson \\ Accessibility Research Centre \\ University of Teesside \\ United Kingdom \\ +44 (0) 1642382656 \\ E.Pearson@tees.ac.uk
}

\author{
Dr Steve Green \\ Accessibility Research Centre \\ University of Teesside \\ United Kingdom \\ +44 (0) 1642382670 \\ S.J.Green@tees.ac.uk
}

\begin{abstract}
Accessibility simulations can give an understanding of the effect a disability may have on the way students access online materials. This paper briefly describes the evaluation of a prototype set of accessibility simulations. The purpose of the prototype was to establish the specification for a second, revised version, which would incorporate the simulation into a learning activity that could be used in Continuing Professional Development (CPD) training for academics. The cognitive overload simulation - part of the second application - has been developed and is subsequently described in detail. In conclusion, this paper discusses planned evaluation of this and similar simulations as an awareness raising tool in workshops for academic staff in Higher Education.
\end{abstract}

\section{Categories and Subject Descriptors}

K.3.1 [Computers And Education]: Computer Uses in Education - Computer-assisted instruction.

I.6.m [Simulation and Modeling]: Miscellaneous.

\section{General Terms}

Design, Human Factors, Experimentation.

\section{Keywords}

Accessibility, Simulations, Disability Awareness, Inclusive Learning, Continuing Professional Development.

\section{INTRODUCTION}

Simulations have already been used successfully in staff development workshops to instill some empathy for the academics with the disabled student experience, help them understand the problem of access and motivate them to adopt new teaching practices [5]. Feedback, however, from previous evaluations indicate that simulations designed specifically to reflect the educational context are required [4]. This research aims to explore the motivational aspects of simulations in education and their ability to support professional development

Copyright is held by the author/owner(s).

ASSETS'08, October 13-15, 2008, Halifax, Nova Scotia, Canada

ACM 978-1-59593-976-0/08/10. activities for staff involved in teaching disabled students [2].

\section{FIRST PROTOTYPE}

A prototype website, comprising a range of appropriate simulations based on interactions with a Virtual Learning Environment (Blackboard) was developed. Blackboard is an established VLE in the academic community, is widely used at the University where the research is based, and it is familiar to staff and students. The purpose of the initial study was to identify the requirements for a second, more sophisticated version, to be used in the context of structured workshops for academic staff, on creating accessible online learning resources. Each one of the developed simulations comprises a task, typical of those students are expected to face in an online course. The software includes simulations for visual disabilities (glaucoma and cataract simulation), motor impairment (mouse control simulation) and cognitive disabilities (distractibility simulation).

The evaluation of the first prototype showed its potential to be used as a learning activity in the CPD context for accessibility awareness raising [3]. The results revealed that although staff appreciated the simulations, some issues were identified which required attention. This led to the creation of a new set of simulations that will more accurately represent the effects of the disability on the user experience in an online learning environment, and will represent a more authentic experience for the academic.

\section{REVISED SIMULATIONS}

In brief, the new application consists of a website which incorporates a new version of the existing simulations, as well as some not included in the first prototype. The new version will be used as a means of not only pointing out the difficulties for disabled students accessing and using web sites and learning resources, but also suggesting ways of alleviating these problems, with the aim of improving inclusion of disabled students in Higher Education. The simulated disabilities have been chosen to reflect the most common disabilities in HE according to the Higher Education Statistics Agency (HESA) [1].

\subsection{New Accessibility Simulations}

For the purposes of this application, the simulations are based on interactions with Blackboard. In the first prototype all the simulations were based on interaction with a single module and only the required links were active. Evaluation revealed that this 
did not reflect genuine interaction. The new simulations are based on a number of modules and users are able to click on different links rather than just the ones needed to complete the task. This can improve realism of the simulation. Eight simulations will be incorporated in the web site: Cataract, Glaucoma, Blindness/Screen Reader user, Colour Blindness, Motor impairment, Hearing/Deafness, Distractibility and Dyslexia. Each simulation is divided into three parts. The accessibility simulation itself, help and instructions for the task that the user is asked to undertake, and feedback with examples of how academic staff can improve their online resources to support disabled students.

\subsection{The Website}

The web site interface contains two main parts. The main navigation menu, on the left, and the contents' space to the right of the main menu. When the user is browsing one of the main categories (i.e. Cognitive, Motor and Visual Disabilities) the contents' space provides information about the simulated disabilities and how it might affect one's interaction with the computer.

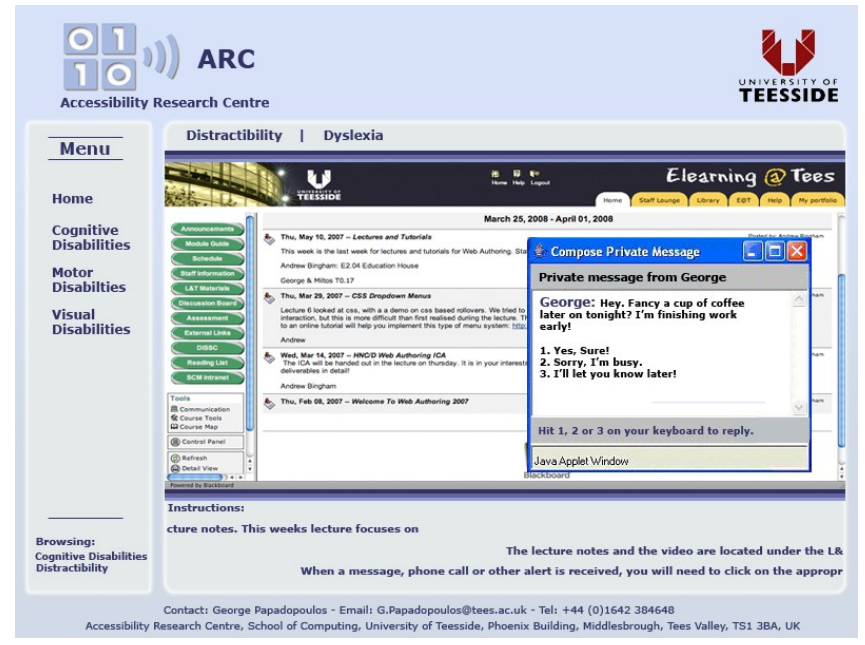

Figure 1: Messages to distract the user

The text, informing the user which part of the web site they are browsing is located at the bottom of the main menu. The screenshot above reveals the location of the secondary menu. In this case, the subcategories of Cognitive Disabilities are Distractibility and Dyslexia and appear on the top of the space provided for contents. The contents' space is divided into three parts when the user is accessing the simulation (Figure 1). The middle and largest space is where the simulation loads. The bottom part displays help and instructions on the task that the user is asked to undertake. Help and instructions are available to users while they are interacting with the simulation. The website has been developed to be accessible and has been checked against the World Wide Web Consortium's (W3C) Web Content Accessibility Guidelines (WCAG).

\subsection{Distractibility Simulation}

For the purposes of this paper an overview of the Distractibility Simulation (cognitive overload) will be presented. Examples of simulations designed specifically to demonstrate the effect of cognitive disabilities in one's interaction with the computer are limited compared to other disabilities. In addition, demonstrating that effect is more difficult and is harder for people to comprehend. Cognitive overload is a feature of many cognitive disabilities, such as autism and dyslexia, and therefore, a characteristic of many students in HE.

In order to distract the users, a virtual chat metaphor is employed. Blackboard offers an embedded chat function, the Virtual Classroom. While interacting with the simulation, a chat window appears occasionally on the screen, informing users about an incoming message. The users are required to reply to the message before moving on to complete the task. To further increase the cognitive load, the simulation includes ambient environmental sounds (crowd noise and voices). Users also experience slow site navigation and are not offered clear instructions on what the task is to complete the activity. This information appears periodically at the space provided, taking the form of rolling text. The effect of these distractions is to split the learner's attention and increase extraneous cognitive load, in order to provide an opportunity for users to encounter the learning experience, in a way that a student with cognitive disability might. A screenshot of the Distractibility Simulation is shown above (Figure 1).

\section{CONCLUSIONS AND FURTHER WORK}

As this new simulation is part of an ongoing study, the newly developed software will be used in the context of structured workshops for academic staff on raising accessibility awareness and creating accessible online learning resources. Their effectiveness as a motivational and awareness raising tool will be tested through evaluations with the workshop participants. In addition, the simulations will be made available to disabled students who will be invited to evaluate the extent to which the prototype simulates accurately their experience with online learning materials. The simulations will also be made available on the Accessibility Research Centre's web site for download, use and evaluation by the academic community.

\section{REFERENCES}

[1] Higher Education Statistics Agency. 2006 - 2007 UK Domiciled Higher Education Students by Disability. Available Online:

http://www.hesa.ac.uk/dox/dataTables/studentsAndQual ifiers/download/disab0607.xls [Accessed 02/05/2008]

[2] Papadopoulos, G. and Pearson, E. 2007. Accessibility Awareness Raising and Continuing Professional Development: The Use of Simulations as a Motivational Tool. Association for Learning Technology Newsletter (ALT-N). Retrieved 17/04/2008, from http://newsletter.alt.ac.uk/e article000735502.cfm

[3] Papadopoulos, G., Pearson, E. and Green, S. 2007. A Preliminary Investigation into the Motivational Aspects of Accessibility Simulations. In Proceedings of the Association for Learning Technology Conference (Nottingham, UK, 2007).

[4] Pearson, E. and Koppi, T. 2003. Developing Inclusive Practices: Evaluation of a Staff Development Course in Accessibility. Australian Journal of Educational Technology. 19, 3, 275-292. 
[5] Pearson, E. and Koppi, T. 2006. A Pragmatic and Strategic Approach to Supporting Staff in Inclusive Practices for Online Learning. In Proceedings of the Australasian Society for Computers in Learning in Tertiary Education (Sydney, Australia, 2006). 